\title{
An Innovative Community-based Model for Improving Preventive Care in Rural Counties
}

\author{
Zsolt J. Nagykaldi, PhD, Dewey Scheid, MD, Daniel Zhao, PhD, Bhawani Mishra, \\ and Tracy Greever-Rice, $P h D$
}

Objective: This quasi-experimental pilot study aimed to implement and evaluate a sustainable, rural community-based patient outreach model for preventive care provided through primary care practices (PCPs) located in a rural county in Oklahoma. A Wellness Coordinator (WC) working with PCPs, the county health department, the county hospital, and a health information exchange (HIE) organization helped county residents receive evidence-based preventive services.

Methods: The WC used a community wellness registry connected to electronic medical records via HIE and called patients at the county level based on PCP-prioritized and tailored protocols. The registry flagged patient-level preventive care gaps, tracked outreach efforts, and documented the delivery of preventive services throughout the community. Return on investment (ROI) for prioritized preventive services was estimated in participating organizations.

Results: Six of the 7 PCPs in the county expressed interest in the project. Three of these practices fully implemented the 1-year outreach program starting in mid 2015. The regional HIE supplied periodic data updates for 9138 county residents to help the coordinators address care gaps using the community registry. A total of 5034 outreach calls were made by the WC in the first year and 7776 prioritized recommendations were offered when care gaps were detected. Of the 5034 distinct patients who received a call, 1146 (22\%) were up to date on all prioritized services, whereas 3888 (78\%) were due for at least 1 of the selected services. Health care organizations in the county significantly improved the delivery of selected preventive services (mean increase, $35 \%$ across 10 services; $P=.004$; range, $3 \%$ to $215 \%$ ) and realized a mean $\mathrm{ROI}$ of $80 \%$ for these services (range, $32 \%$ to $122 \%$ ). The health system that employed the WC earned an estimated revenue of $\$ 52,000$ realizing a $40 \% \mathrm{ROI}$ for the coordinator position.

Conclusions: Although more research is needed, our pilot study suggests that it may be feasible and cost effective to implement an innovative, county-level patient outreach program for improving preventive care in rural settings. ( $\mathrm{J}$ Am Board Fam Med 2017;30:583-591.)

Keywords: Community Health, Outreach, Prevention, Primary Care, Wellness Coordinatorh

Optimizing the delivery and receipt of 6 key preventive measures is estimated to save approximately 100,000 lives a year in the United States (aspirin prophylaxis; smoking cessation; influenza vaccina-

This article was externally peer reviewed.

Submitted 7 February 2017; revised 7 February 2017; accepted 13 March 2017.

From the College of Medicine, Department of Family and Preventive Medicine, University of Oklahoma Health Sciences Center, Oklahoma City, OK (ZJN, DS); College of Public Health, Department of Biostatistics and Epidemiology, University of Oklahoma Health Sciences Center, Oklahoma City (DZ); Division of Applied Social Sciences, Office of Social and Economic Data Analysis, University of Missouri, Columbia, MO (BM, TGR)

Funding: The study was funded by a research grant from the Agency for Healthcare Research and Quality (AHRQ) Grant 1R18HS023237. tion; and colorectal, breast, and cervical cancer screening). ${ }^{1}$ However, primary care practices (PCPs) continue to fall short of providing preventive services to all patients who could benefit. ${ }^{1-4}$ There are several reasons for this problem. Yarnall and colleagues ${ }^{5,6}$ calculated that it would take primary care clinicians over 21 hours per day to provide high-evidence primary and secondary preventive services recommended by the US Preventive

Conflict of interest: none declared.

Corresponding author: Zsolt J. Nagykaldi, PhD, College of Medicine, Department of Family and Preventive Medicine, University of Oklahoma Health Sciences Center, $900 \mathrm{NE}$ $10^{\text {th }}$ Street, Oklahoma City, OK 73104 (E-mail: znagykal@ ouhsc.edu). 
Services Task Force (USPSTF) and tertiary preventive care to patients with chronic illnesses recommended by disease-specific guidelines. The diversity of electronic health records that are not interoperable and the decreasing margin of resiliency of PCPs, especially in rural areas, may also contribute to suboptimal care delivery. ${ }^{7,8}$

More recently, hospitals have become more engaged in prevention, providing immunizations for patients with pneumonia, smoking cessation counseling, referrals for tobacco-related illnesses, and low-dose aspirin or $\beta$ blockers for patients with heart attacks. County health departments (CHDs) are also committed, particularly to primary prevention. However, large segments of the population rarely visit hospitals or health departments and only approximately half of recommended preventive services are received by the adult population in the United States. ${ }^{9}$

Efforts of these 3 potential partners (PCPs, hospitals, and CHDs) are often not well aligned. For example, PCPs are frustrated by the adverse health effects of physical inactivity and obesity, perceiving that these are public health problems, which they are expected to address. They are also frustrated that when many low-income children are immunized at the CHD, they may not present to their well-child visits. A significant portion of reimbursements for the time and resources required by PCPs to make sure patients get referred screening tests go to the subspecialists and hospitals. When immunizations are given in the hospital or CHD, the information may often not make it back to the PCP records. CHDs have trouble getting practices and hospitals to use immunization registries, and they would like to see more patients referred to them for immunizations.

Rural populations suffer both from poorer health and decreased access to quality care than their urban and suburban counterparts. ${ }^{10}$ They are older on average and have lower income, less education, and higher rates of smoking and obesity. Rates of hypertension, diabetes, and cardiovascular disease are also higher in rural counties. ${ }^{11}$ Despite greater health care needs, fewer than half as many physicians per capita work in rural counties as in urban and suburban counties in Oklahoma. ${ }^{12}$ Therefore, new models of health care delivery in rural America are needed.

A 2012 Institute of Medicine (IOM) report entitled, "Integrating Primary Care and Public Health," indicated renewed interest in collaborative models. ${ }^{13}$
The IOM Committee pointed to the need for "neutral convener" organizations that bring together primary care and public health professionals. Hospitals must adjust their business plans to the changing epidemiology of illness and efforts to reduce admissions, early readmissions, and emergency department visits. Nonprofit hospitals that accept Medicare are now required to construct and carry out community health improvement plans. Accountable care organizations are forging new relationships between PCPs and hospitals.

Based on these trends and needs, we initiated a 4-year study to implement, evaluate, and disseminate a sustainable, rural community-based patient outreach model to improve preventive care provided through 20 PCPs located in 3 rural counties in Oklahoma. Wellness Coordinators (WCs) working with PCPs, CHDs, local hospitals, and Health Information Exchange (HIE) organizations helped county residents receive evidence-based preventive services. A quasi-experimental implementation pilot study that was part of the 4-year initiative and what we describe in this article evaluated the impact of the community-level preventive services outreach program in the first of the 3 consecutively engaged rural counties.

\section{Methods}

\section{Partnerships and Program Design}

Collaborating with the academic study team, the regional health system-owned county hospital hired a full-time WC in the pilot intervention county. To alleviate the financial risk of the initial experiment, the WC's first-year salary was paid through a research grant from the Agency for Health care Research and Quality (AHRQ). The hospital contract delineated that after the first year, the burden of WC support would transition to the hospital, contingent on sufficient revenues generated from some of the preventive services referred from participating practices (eg, colonoscopies, mammograms, and bone density scans). Six of the 7 practices operating in the county were recruited into the study in mid 2014. Three of these fully implemented the outreach program starting in mid 2015, whereas the other 3 achieved partial implementation. They received a full-time Practice Enhancement Assistant (PEA), also called practice facilitator, ${ }^{14,15}$ to help them prioritize 3 to 4 preventive services, create patient outreach protocols, and ac- 
celerate the implementation of improved care delivery processes. In addition to practice-based priorities, participating health care organizations chose to focus on smoking cessation and physical activity improvement through consensus building.

The collaboration was overseen by a local nonprofit County Health Improvement Organization (CHIO) that acted as an "umbrella" to ensure transparency, create mutual trust, and align the activities of individual organizations with the goals of the community. The regional HIE entered into a service contract with the study team and also with health care organizations in the county to supply interoperable clinical records (Continuity of Care Documents) for county residents whose primary care home was in participating practices. Data on these individuals included information from the county, and also from regional hospitals, diagnostic laboratories, imaging facilities, and specialty clinics. These data connections and the quality of data have evolved significantly in the course of the pilot project. The CHD was also engaged and participated by providing immunizations and facilitating the community health assessment process. A map of the county partnership and information flow are represented in Figure 1.
Through meetings with the CHIO and by leveraging their connections in the community, the project was advertised as a health and wellness campaign for the county ("Healthier Together Campaign") via media outlets, community meetings, schools, and faith-based organizations. The project team also attempted to bring additional, scarce resources into the county (eg, counseling services, diabetes train-the-trainer programs, and screening opportunities) and worked with state and national organizations to establish more effective tobacco-cessation services by linking patients directly to the Oklahoma Tobacco Help Line.

\section{Community Intervention}

Outreach calls were tracked systematically by a secure community registry (Preventive Services Reminder System) that the investigators developed earlier. ${ }^{16}$ The registry included a preventive care forecasting function that recommended person-tailored services based on HIE data, USPSTF guidelines (A and $\mathrm{B}$ ), personal risk factors, and seasonal variations of need. The WC called county residents in their birth month annually, based on overlaps between personal care gaps flagged by the registry and a list of high-priority services determined by

Figure 1. The "Healthier Together" Project County Partnership. CHIO, County Health Improvement Organization; HIE, Health Information Exchange; PCP, Primary Care Physician/Provider; PEA, Practice Enhancement Assistant; PSRS, Preventive Services Reminder System; Pts, Patients; WC, Wellness Coordinator.

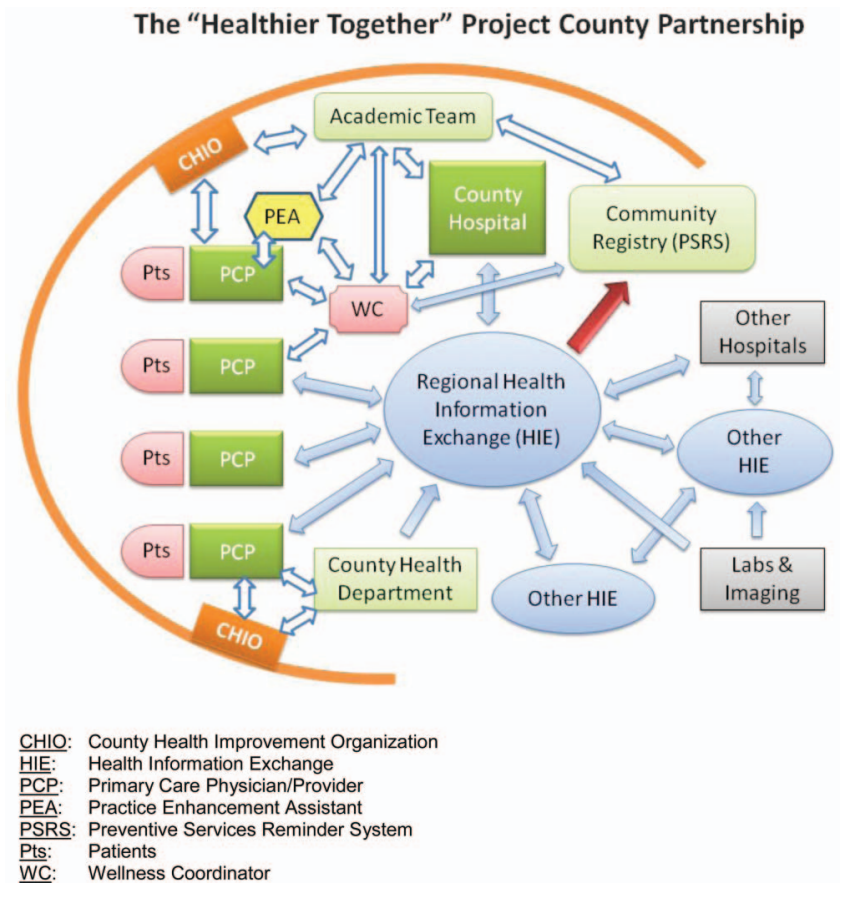


the practices and the local community working through the CHIO. Calls followed practice-tailored protocols and organization-approved scripts with branching logic.

\section{Outcomes and Analyses}

Rates of preventive service delivery for selected services were calculated for a preimplementation baseline year and for the intervention year for each participating practice and the hospital using a combination of population-level HIE records and practice-level patient records and by generating representative samples of patients who were due for specific services based on USPSTF guidelines each year. Pre- and postimplementation service rates were compared as correlated proportions using the McNemar's test.

The investigators partnered with the [University of Missouri] Office of Social and Economic Data Analysis to guide econometric data collection and analyses. To conduct return-on-investment (ROI) analyses, the team created an analytic database organized by intervention and provider type. Data collected across analytic dimensions were normed by prioritizing the use of common codes and billing data to calculate revenue as well as standard cost indicators, such as salaries and benefits, materials and supplies, and overhead for each priority preventive service. To establish a baseline, financial data were also collected for a year preceding the intervention in each practice and for each service. The discount rate for the base year was applied to intervention year projections and additional organizational investments were also considered. Incremental costs and revenues were calculated for the delivery of selected preventive services by practice and compared with those in the baseline year to calculate ROI estimates.

The PEA took detailed field notes on observations pertaining to the implementation process in each practice and the study team maintained project-level progress notes on the development of the program. CHIO stakeholder meeting agenda and minutes were also collected to record communitylevel discussions on the prevention outreach program, including meeting dates, topics discussed, barriers identified, and interventions suggested or tried. These documents were later mined using content analytic techniques to construct a rich description of the community environment, the participants, emerging facilitators and barriers, solutions, and lessons learned from building the experimental system in the county.

The study was approved and monitored by the [University of Oklahoma Health Sciences Center] Institutional Review Board.

\section{Results}

In a 12-month period starting in June 2015, the regional HIE supplied monthly data updates for 9138 patients who were seen by participating practices. A demographic profile of the patient population reached by WCs is provided in Table 1 . On average, 6 evidence-based preventive recommendations were suggested per person by the community registry. Of these, typically, 1 to 3 recommendations were offered by the WC per person that overlapped with practice or community priorities. Across all participating practice sites, 10 preventive services were

Table 1. Demographics of the Patient Population Reached by Oklahoma County Wellness Coordinators During the Intervention Period

\begin{tabular}{lcc}
\hline Demographic Parameter & Outreach Effort Outcome & County Population Reference \\
\hline Number of Individuals & 9,138 & $\sim 15,000$ \\
Age (years), mean (SD) & $38(21)$ & Mean: 38 \\
Sex (female) & $50.5 \%$ & $52.1 \%$ \\
Race/ethnicity & Insufficient data available in health records & $84 \%$ White \\
& & $12.4 \%$ Hispanic/Latino \\
Spatial distribution of population & & $30 \%$ \\
$\quad$ (by town) & $62 \%$ & $15 \%$ \\
Town A & $8 \%$ & $3 \%$ \\
Town B & $7 \%$ & $\$ 37,000$ \\
Town C & Data not available in health records
\end{tabular}


offered, including: colonoscopy, mammography, bonedensity scans, diabetes checkups, hemoglobin A1c (hbA1c) management, flu shots, pneumococcal vaccinations, physical activity counseling, smoking-cessation counseling, and well-child visits.

\section{Quantitative Outcomes}

A total of 5034 outreach calls were made in the first year and 7776 prioritized recommendations were offered based on care gaps. The WC spent approximately 660 hours on the phone performing direct outreach activities. Of the 5034 patients who received 1 call over the implementation year, 1146 (22\%) were up to date on all prioritized services, whereas 3888 (78\%) were due for at least 1 service. In the implementation year, $35 \%$ more preventive services were delivered to patients in the county across 10 services compared with the baseline year, ranging from a $3 \%$ to a $215 \%$ increase in the delivery of services $(P=.004)$. Improvements by service are summarized in Table 2. Practices realized a mean ROI of $80 \%$ on these services ranging from $32 \%$ to $122 \%$. The health system that owned the county hospital and employed the WC earned $\$ 52,000$ in estimated revenues from improvements in service delivery compared with a baseline year, which offset the cost of the WC and generated a $40 \%$ ROI for the hospital on the WC's employment cost. Seventy-five percent of the hospital's revenue came from an increase in the provision of 3 referred screening tests, including colonoscopies, mammograms, and bone-density scans, whereas another $25 \%$ came from laboratory tests, immunizations, and well-child visits provided by the hospital's outpatient clinic.

\section{Qualitative and Process Outcomes}

Based on these results, the county hospital and its health system leadership decided to continue funding the WC position from their own resources after the first year. However, achieving this outcome was not easy. The study team had to spend an excessive amount of time with negotiating and executing contracts and agreements, struggling with technology vendors to establish data interfaces in a timely fashion, and working with competing health systems. In particular, legal agreements that satisfied multiple entities regarding health data protection, ensuring proper patient attribution to practices in HIE records, and technology vendor idiosyncrasies

Table 2. Rates of Delivering Preventive Services Before and After Outreach Program Implementation, Grouped by Service Type and Care Delivery Domain

\begin{tabular}{|c|c|c|c|c|}
\hline Preventive Service Type & $\begin{array}{l}\text { Baseline } \\
\text { Service Rate* }\end{array}$ & $\begin{array}{l}\text { Intervention } \\
\text { Service Rate* }\end{array}$ & $P$ & $\begin{array}{l}\text { Share of Total } \\
\text { ROI }^{\dagger}\end{array}$ \\
\hline \multicolumn{5}{|l|}{ Primary care practice domain } \\
\hline Smoking cessation counseling & $33 \%$ & $71 \%$ & $<.01$ & $14 \%$ \\
\hline Adult immunizations (influenza and pneumococcal vaccine) & $63 \%$ & $78 \%$ & $<.05$ & $3 \%$ \\
\hline $\begin{array}{l}\text { Diabetes management (diabetes checkup visits \& HbA1c } \\
\text { measurement) }\end{array}$ & $48 \%$ & $75 \%$ & $<.01$ & $18 \%$ \\
\hline Well child visits & $51 \%$ & $60 \%$ & $<.05$ & $13 \%$ \\
\hline Physical activity counseling & $27 \%$ & $38 \%$ & $<.01$ & $14 \%$ \\
\hline All practice-based services & $44 \%$ & $64 \%$ & $<.01$ & $62 \%$ \\
\hline \multicolumn{5}{|l|}{ County hospital and health system domain } \\
\hline Colonoscopy (referred) & $38 \%$ & $43 \%$ & $.07^{\ddagger}$ & $31 \%$ \\
\hline Mammography (referred) & $55 \%$ & $63 \%$ & $<.05$ & $6 \%$ \\
\hline Bone density screening (referred) & $24 \%$ & $30 \%$ & $<.05$ & $1 \%$ \\
\hline All referred services & $39 \%$ & $45 \%$ & .05 & $38 \%$ \\
\hline \multicolumn{5}{|l|}{ County healthcare domain } \\
\hline All services combined & $42 \%$ & $57 \%$ & $<.01$ & $100 \%$ \\
\hline
\end{tabular}

*Service rates were measured by combining HIE record analyses and medical record abstractions. HIE records and health system-level service reports were essential to estimate the rate of referred services (mammography, colonoscopy, and bone density screening).

${ }^{\dagger}$ Total ROI includes all returns generated across the project, including all organizations and services.

${ }^{\ddagger}$ Although this trend did not reach statistical significance across the county, there was an increase in the absolute number of colonoscopies that were referred to the health system that employed the wellness coordinator during the intervention period compared to the baseline period. Most of these patients received a call from the wellness coordinator. 
required substantial unanticipated time, effort, and resources. Numerous workarounds and many adaptations of the original project plan had to be instituted to respond to emerging challenges and the constantly changing health care environment. Health information technology vendors requested the payment of various, sometimes arbitrary fees for establishing and maintaining data interfaces, most of which were unknown at the time of project initiation. Service fees showed a 5-to-10-fold variation across vendors and there was a significant difference between vendors in their willingness to negotiate the cost.

The ongoing turmoil in small, rural PCPs, even in 1 year and in 1 county, was remarkably high, including disruptive ownership changes, electronic medical record replacements, retirement or death of providers, loss and turnover of clinicians and key staff, and significant financial difficulties. Most of these rural practices were new to systematic quality improvement, which required extra PEA time to build some organizational capacity for change. These challenges resulted in a 6-month implementation delay compared with the original project timeline.

Despite the struggle, all practices benefited from participating by improving the delivery of preventive care, receiving new resources that helped them reach meaningful use requirements, gaining access to care, quality improvement support, and generating ROI on specific services. The county benefited from instituting a community-wide wellness outreach program, attracting funding and health care resources into the county (eg, CHIO support), incentivizing the local hospital to invest into scarce services (eg, colonoscopy), and linking the community to a statewide network of professional health care expertise through the study team.

\section{Discussion}

Although more research is needed, our demonstration project suggests that it may be feasible and cost effective to implement an innovative, county-level patient outreach program for improving preventive care in rural settings. Through partnerships with various local health organizations, a shared infrastructure can be built that may accelerate the improvement of population health in rural communities.

The purpose of the study was to create an experimental rural environment in which at least some of the misaligned incentives for promoting and providing prospective care may be corrected. Although PCPs provide the majority of the work to promote and deliver preventive services, historically, they have not received commensurate support for these activities, while subspecialists have benefitted considerably from referrals originating from primary care. Capturing and redistributing some of these revenues at the community level to support highly needed health care services (eg, patient outreach) may help align the participation of community partners, and, as the results suggests, it may also facilitate improved population health in rural settings more effectively.

Employing the WC by an entity that was most likely to benefit and making sure that the generated value remained transparent and accessible to the community through the CHIO helped trust building and gathering otherwise less willing partners around the table. The partners understood that while the WC called on behalf and with the instructions of individual clinicians, her work was coordinated and supported at the county level for better effectiveness and scale of economy. Ongoing networking of county partners through the CHIO made it possible to align the efforts of each partner with community goals. In fact, 1 of the criteria for selecting the intervention county was the presence of an active CHIO, which is the building block of the primary health care extension system in Oklahoma. ${ }^{17-19}$

Although grant funding for the work of the PEA was continued beyond the pilot project, practices were able to build capacity to improve care delivery processes, which allowed them to taper facilitation support over the intervention period. This is consistent with the practice facilitation literature. ${ }^{20}$ However, initial assistance was essential to help practices select high-priority preventive services, design outreach protocols, enhance their prevention workflow, learn how to track their progress, and align their activities with the goals of the community.

Perhaps the greatest challenge of this study was to work with health information technology vendors that provided electronic medical record access and data exchange services across heterogeneous organizations. This study and other large national initiatives (eg, AHRQ's ongoing EvidenceNOW project) have consistently found that a significant mismatch exists between the intent of evolving fed- 
eral programs for health technology development and what actually transpires in the field. The perception of many health care providers is that the certification of health information technology (IT) products and services may not mean much in reality, due to vendor attempts to circumvent the requirements by preventing meaningful access to health information ("data blocking"), or providing some of the required features to users only if they pay more. These discrepancies burden especially small, independent rural practices that will have to bridge technology gaps with their own resources. A much stricter regulatory oversight should be in place to prevent these serious problems and effective means should be offered to providers to request remediation when products and services fall short of what was advertised or promised. Numerous technical and legal aspects of interoperability remain ill-conceptualized, may be constantly changing or are not clearly defined. Given that quality data are in the center of modern health care, there is an urgent need to address this situation on a national scale. Problems with the accessibility and quality of health care data may undermine large-scale efforts to improve health care in the United States. Driven by our team's determination to advance data access and interoperability, the quality and depth of integrated patient records continued improving throughout the study, albeit not without major frustrations. Table 3 lists the most frequent data availability and quality problems seen in this study.

The study also suggests that legal professionals employed by health care organizations may need specific training and experience in working with community-wide, multi-stakeholder initiatives. The study team invested a substantial amount of time to advance innovations in organizations that formerly adopted an aggressive legal risk-minimizing approach, which made it more difficult to change the status quo. Our findings demonstrate the need for experiential training of legal and administrative personnel on best practices for supporting health

Table 3. Types, Frequency and Consequences of Problems with the Availability or Quality of Health Information and Solutions Implemented in the Healthier Together Pilot Study

\begin{tabular}{|c|c|c|}
\hline $\begin{array}{l}\text { HIE Data Quality } \\
\text { Problem Type }\end{array}$ & $\begin{array}{c}\text { Frequency of Data Quality Problem in } \\
\text { HIE Records }\end{array}$ & $\begin{array}{c}\text { Consequences of Data Quality Problem \& } \\
\text { - Mitigation Strategies }\end{array}$ \\
\hline $\begin{array}{l}\text { Patient is not attributed } \\
\text { to a PCP (no PCP } \\
\text { listed) }\end{array}$ & Decreased from $94 \%$ to $80 \%$ over the pilot & $\begin{array}{l}\text { Services \& quality metrics can't be linked to } \\
\text { PCP Additional patient matching using } \\
\text { EHR data }\end{array}$ \\
\hline $\begin{array}{l}\text { Low actionable* data } \\
\text { contribution from } \\
\text { PCPs }\end{array}$ & Decreased from $89 \%$ to $66 \%$ over the pilot & $\begin{array}{l}\text { Less information on PCP services }>\text { Improving } \\
\text { data interfaces \& manual extractions from } \\
\text { EHRs }\end{array}$ \\
\hline $\begin{array}{l}\text { Missing or wrong } \\
\text { patient phone } \\
\text { numbers }\end{array}$ & Decreased from $49 \%$ to $38 \%$ over the pilot & $\begin{array}{l}\text { Patient reach barriers } \rightarrow \text { Better documentation } \\
\& \text { additional data extraction from billing } \\
\text { records }\end{array}$ \\
\hline $\begin{array}{l}\text { Risk factor rate lower } \\
\text { or higher than } \\
\text { expected }^{\dagger}\end{array}$ & $\begin{array}{l}\text { Smoker }\left(5 \% \text { vs. } 16 \%^{\ddagger}\right) \\
\text { Diabetic }\left(21 \% \text { vs. } 10 \%^{\ddagger}\right)\end{array}$ & $\begin{array}{l}\text { Inaccurate care gap predictions }>\text { Better chart } \\
\text { documentation and calibrating data interfaces }\end{array}$ \\
\hline $\begin{array}{l}\text { Preventive service rates } \\
\text { are lower than } \\
\text { expected }^{\dagger}\end{array}$ & $\begin{array}{l}\text { Mammography ( } 5 \% \text { vs. } 11 \%^{\ddagger} \text {; increased to } \\
13 \% \text { over time) }\end{array}$ & $\begin{array}{l}\text { Inaccurate care gap predictions }>\text { Better chart } \\
\text { documentation and calibrating data interfaces }\end{array}$ \\
\hline $\begin{array}{l}\text { Race or ethnicity } \\
\text { information not } \\
\text { available }\end{array}$ & $\begin{array}{l}\text { About } 98 \% \text { to } 99 \% \text { of records (remained } \\
\text { unchanged) }\end{array}$ & $\begin{array}{l}\text { Less tailored care recommendations Improve } \\
\text { documentation of race in patient chart }\end{array}$ \\
\hline $\begin{array}{l}\text { Skewed data } \\
\text { contribution among } \\
\text { organizations }\end{array}$ & $\begin{array}{l}30 \% \text { of HIE records are over-concentrated } \\
\text { in SE of county }\end{array}$ & $\begin{array}{l}\text { Some organizations dominate as data source } \\
\text { - Oversample records in northern county } \\
\text { region }\end{array}$ \\
\hline
\end{tabular}

EHR, Electronic Health Record; HIE, Health Information Exchange (patient records aggregated regionally); PCP, primary care practice.

*Actionable data include health risk factors (e.g., smoking status), reports, and laboratory findings pertinent to prevention, and history of preventive services. Low-value data include administrative visit information and free text notes that often "bloat" interoperable records causing excessive transmission and processing times.

${ }^{\dagger}$ Rate means the frequency of the occurrence of health risk factors or preventive services in HIE datasets relative to the known prevalence of these factors in the population.

${ }^{\ddagger}$ State of the State's Health Report 2014, Oklahoma State Department of Health. 
care innovations while remaining confident that they can protect their institution.

Similarly, academic institutions tend to support basic science and traditional clinical research, but they may not always have the infrastructure to facilitate patient and community-centered research that includes nonprofit entities, community organizations, and under-resourced stakeholders. Long administrative and contracting procedures, delays in payments, issues with perceiving conflicts of interest when faculty are also active in the community, and the complexity of navigating research processes often represent significant barriers for community partners to interface with research.

Lessons learned from this pilot study will allow our team to extend the implementation of the wellness outreach program to 2 other rural counties in Oklahoma. Lessons included the approach to convening the health community of an entire county around prevention; setting up shared procedures, service contracts and legal agreements; building a community-level data infrastructure; establishing a county-wide patient outreach program; and aligning outreach operations with financial benefits generated across organizations. At the end of 2016, a second county has initiated the program with improvements and adaptations and the implementation is being planned in a third county. The outcomes of the project will be used to create and disseminate a toolkit that will help others replicate the outreach program in their environment.

\section{Conclusions}

Although disparate health care entities in rural counties can be brought together to improve the health of the community through an organized wellness outreach program, the fragmentation and misalignment of the health care system makes it challenging to build such collaborations across the boundaries of organizations and their diverging interests. However, when these partnerships are established successfully, they may be able to achieve and economically sustain community-wide health improvement by creating a win-win situation for all partners.

The authors express their sincere appreciation to primary care practices, community partners, technology service providers, and patients who participated in this study. Their support has been essential to the success of the community wellness program.
To see this article online, please go to: http://jabfm.org/content/ 30/5/583. full.

\section{References}

1. Partnership for Prevention. Preventive Care: A national profile on use, disparitities, and health benefits. Washington DC: National Commission on Prevention Priorities; 2007.

2. Chowdhury P, Balluz L, Town M, et al. Surveillance of certain health behaviors and conditions among states and selected local areas-Behavioral Risk Factor Surveillance System, United States, 2007. MMWR Surveill Summ 2010;59:1-220.

3. McGlynn EA, Asch SM, Adams J, et al. The quality of health care delivered to adults in the United States. N Engl J Med 2003;348:2635-45.

4. Pham HH, Schrag D, Hargraves JL, et al. Delivery of preventive services to older adults by primary care physicians. JAMA 2005;294:473-81.

5. Yarnall KS, Pollak KI, Ostbye T, et al. Primary care: Is there enough time for prevention? Am J Public Health 2003;93:635-41.

6. Yarnall KS, Ostbye T, Krause KM, et al. Family physicians as team leaders: "Time" to share the care. Prev Chronic Dis 2009;6(2):A59.

7. Krist AH, Beasley JW, Crosson JC, et al. Electronic health record functionality needed to better support primary care. J Am Med Inform Assoc 2014;21:764-71.

8. Ruhe M, Gotler RS, Goodwin MA, et al. Physician and staff turnover in community primary care practice. J Ambul Care Manage 2004;27:242-8.

9. Shires DA, Stange KC, Divine G, et al. Prioritization of evidence-based preventive health services during periodic health examinations. Am J Prev Med 2012;42: 164-73.

10. Office of Rural Health Policy (ORHP). Rural Healthy People 2010_-"Healthy People 2010: A companion document for rural areas": Texas A\&M Health Sciences Center; 2010.

11. Bennett KJ, Bankole O, Probst JC. Health disparities: A rural-urban chartbook. South Carolina: Rural Health Research and Policy Centers; 2008.

12. State of the State's Rural Health. Stillwater OK: Okahoma State University Center for Health Sciences; 2008.

13. Institute of Medicine. Primary care and public healthExploring integration to improve population health. Washinton, DC. Institute of Medicine; 2012 March.

14. Nagykaldi Z, Mold JW, Aspy CB. Practice facilitators: A review of the literature. Fam Med 2005;37:581-8.

15. Nagykaldi Z, Mold JW, Robinson A, et al. Practice facilitators and practice-based research networks. J Am Board Fam Med 2006;19:506-10.

16. Nagykaldi Z, Mold JW. The Role of Health Information Technology in the Translation of Research into Practice: An Oklahoma Physicians Resource/ 
Research Network (OKPRN) Study. J Am Board Fam Med 2007;20:188-95.

17. Grumbach K, Mold JW. A health care cooperative extension service: Transforming primary care and community health. JAMA 2009;301:2589-91.

18. Mold JW. A cooperative extension service for primary care in Oklahoma. J Okla State Med Assoc 2011;104(11-12):414-8.
19. Phillips RL Jr, Kaufman A, Mold JW, et al. The primary care extension program: A catalyst for change. Ann Fam Med 2013;11:173-8.

20. Mold JW, Aspy CB, Smith PD, et al. Leveraging practice-based research networks to accelerate implementation and diffusion of chronic kidney disease guidelines in primary care practices: A prospective cohort study. Implement Sci 2014;9:169. 